\title{
P-Stop Designs for Reducing Electric Field Strength at Implant Edges
}

\author{
Y. Unno, Y. Ikegami, T. Kohriki, S. Terada, K. Hara, K. Yamamura, S. Kamada
}

\begin{abstract}
In designing the radiation-tolerant silicon microstrip sensors, the onset of microdischarge must e expelled above the maximum operation voltage. The difficulty is enhanced in the $n$ in-p sensors, with the existence of $p$-n junction in the $n$-strips and the existence of p-stop structures. The device simulation program enabled to understand the electric fields in detail and to optimize the design to be robust against the microdischarge. The potential of the p-stop is the fundamental. In the common p-stop structures, the narrowest p-stop width has the shallowest potential and generating the least electric field strength (Emax). The potential of the split p-stops near to the n-implant in the combined p-stop does not have a potential closer to that of the $n$-implant. The symmetric location of p-stop has the least Emax. The Emax increases as the strip pitch decreases less than 80 microns but stays the same as the pitch widens larger than 80 microns. The onset voltage of Punch-thru protection (PTP) is governed by the gap between the $n$-implants, $N-N$ gap, even with the existence of p-stop in between.
\end{abstract}

\section{INTRODUCTION}

A silicon semi-conductor position sensitive device called "silicon microstrip sensor" has been used widely in the elementary particle physics experiments, such as the SCT detector of the ATLAS experiment at LHC 0 . The future of LHC is to increase the luminosity by a factor of 10 , the super LHC (SLHC), and to collect data at least by a factor of 5, thus the expected fluence at the SLHC is at least 5 times that of $\mathrm{LHC}$, and is about $1 \times 10^{15} 1-\mathrm{MeV}$ neutron equivalent $/ \mathrm{cm}^{2}$ at about a radius of $30 \mathrm{~cm}$. With this fluence, the full to over depletion of about $300 \mu \mathrm{m}$ thickness is unlikely with a reasonably high bias voltage, e.g., $500 \mathrm{~V}$, the direction of $R \& D$ of the radiation-tolerant silicon microstrip sensor for SLHC is to utilize n-implant strips in p-bulk wafers, so-called "n-in-p" sensor [2].

The most difficult aspect in designing a radiation-tolerant silicon microstrip sensor is to expel the onset voltage of the "micro discharge" higher than the bias voltage required for the operation of the sensor. The microdischarge is the steep increase of the leakage current due to the electron-hole generation at the spots where the electric field strength exceeds the breakdown field that is about $300 \mathrm{kV} / \mathrm{cm}$ in the silicon. The spots can be visualized with the infrared light and an example is shown in Fig. 1.

Manuscript received November 14, 2007. This research was supported by Grant-in-Aid for Scientific Research (A), 20244038.

Y. Unno (e-mail: yoshinobu.unno@kek.jp), Y. Ikegami, T. Kohriki, S. Terada are with the IPNS, High Energy Accelerator Research Organization, KEK, Tsukuba, Ibaraki 305-0801, Japan.

K. Hara is with the Institute of Pure and Applied Sciences, University of Tsukuba, Tsukuba, Ibaraki 305-8571, Japan.

K. Yamamura, S. Kamada are with Solid State Division, Hamamatsu Photonics Co. Ltd., Hamamatsu, Shizuoka 435-0051, Japan.

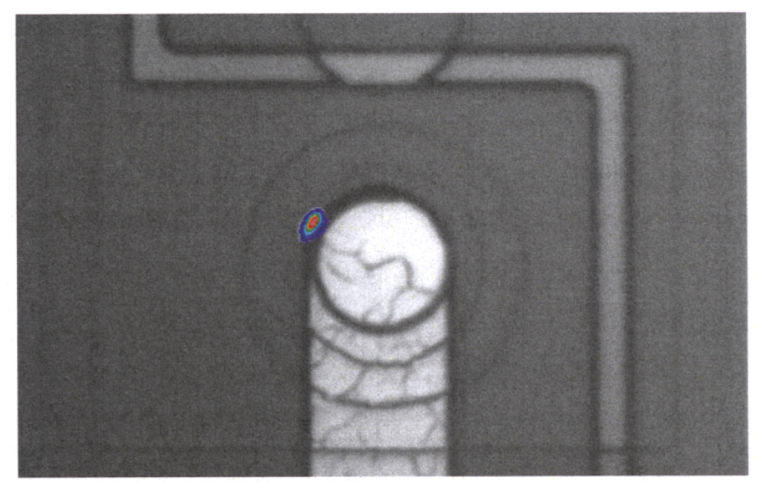

Fig. 1 Hot spot of micro discharge observed with an infrared-sensitive camera, overlaid on the visible light image

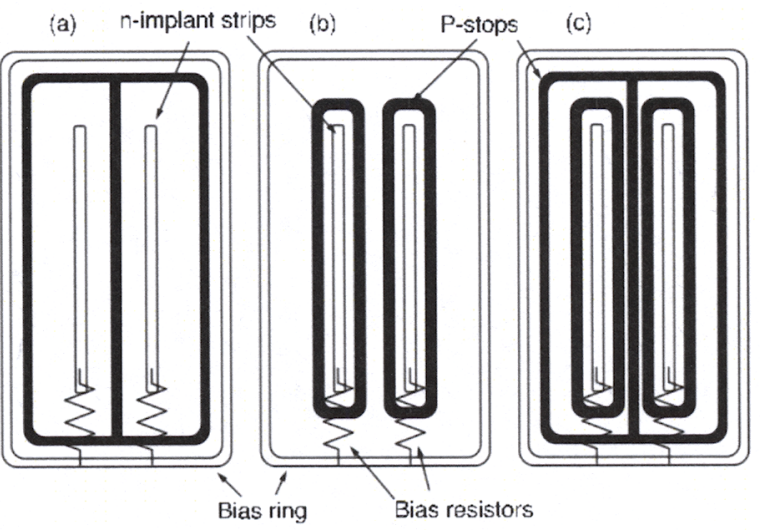

Fig. 2 Representative schematics of p-stop structures: (a) common, (b) individual, and (c) combined

This difficulty is enhanced in the $n$-in-p sensor due to the existence of $p-n$ junction in the $n$-implant strips and the existence of $\mathrm{p}$-stop structures. The p-stop structure is required in the n-side of the silicon sensor. The interface between the silicon bulk and the surface oxide tends to be charged positively. These positive charges attract electrons in the interface, forming the accumulation layer and shorting the nimplant strips. In order to isolate the n-implant strips, this accumulation layer must be interrupted with a p-type surface implantation. One method is to implant p-type ions with a lithography mask. The implant is called "p-stop". The other is to implant p-type ions all over the surface and is called "pspray". Fig. 2 shows a few examples of "p-stop" methods. We present an insight to the electric field in these structures and how to optimize the design of the p-stop structures in order be robust against the microdischarge. 


\section{TECHNOLOGY CAD SimULATION}

The semiconductor industry has developed sophisticated programs, called "Technology CAD (or TCAD)", to simulate the processes and the devices, pioneered by $\mathrm{R}$. Dutton et al. [3]. We used a TCAD program called ENEXSS [4] to simulate the device in $2 \mathrm{D}$ for simplicity although the program has capability of 3D simulation. For the representative p-stop structures whose surface geometries are shown in Fig. 2, the simulations are made for the cross-section between the nimplant strips along the centerline crossing the $n$ - and $p$-stop implantation. The cross-sectional geometry model is shown in Fig. 3. An example of electric field strength in two-dimension (2D) is shown in Fig. 4.

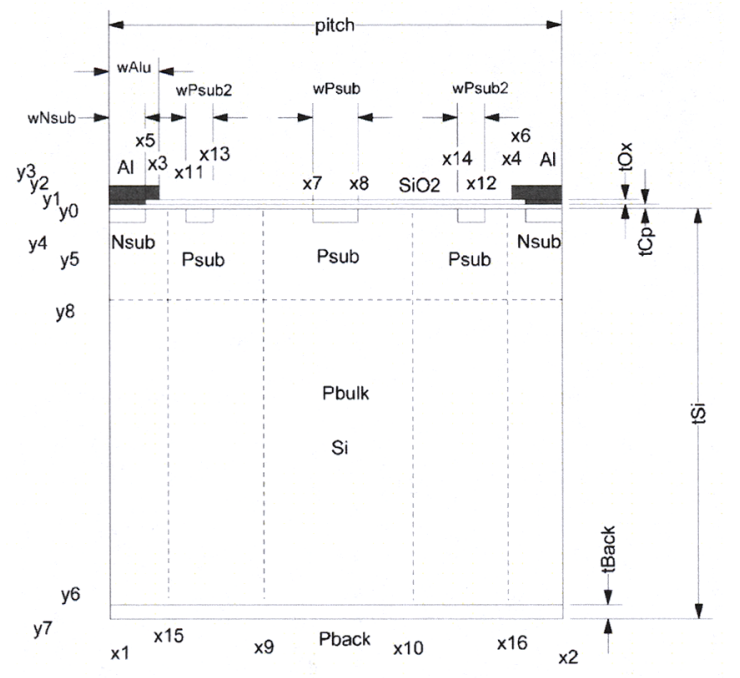

Fig. 3 Geometry model of the TCAD simulation for the p-stop structures. The common p-stop is the one at the center. The rings of individual p-stop are modeled with the p-stops near the n-implant at the edges.

wPsub06_dis.dists0.E

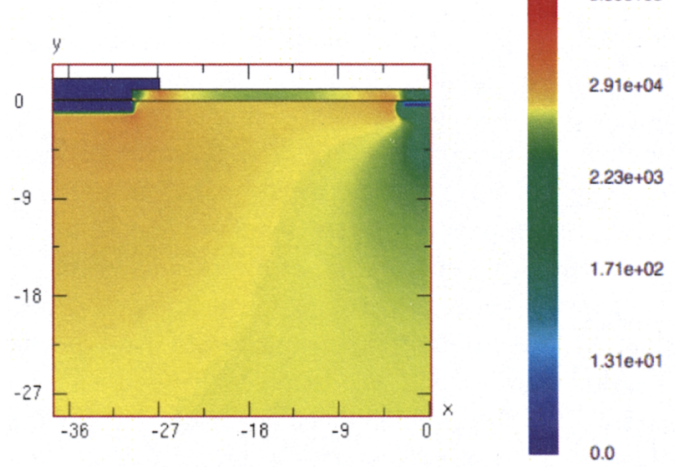

Fig. 4 A 2-dimenstional plot of the electric field strength between the nimplant and the p-stop of the common p-stop structure. High fields are at the edges of the $n$-implant and the $p$-stop. The scale of the left figure is in microns. The scale in the right is $\mathrm{V} / \mathrm{cm}$.
In the simulations, otherwise mentioned, the resistivity of $\mathrm{p}$ bulk was $3 \mathrm{k} \Omega \mathrm{cm}$; the dopant concentration of $\mathrm{n}$-implant (Nsub) $1 \times 10^{14}$ ions $/ \mathrm{cm}^{2}$, the p-stop (Psub) $4 \times 10^{12}$ ions $/ \mathrm{cm}^{2}$ in a depth $(\mathrm{y} 0-\mathrm{y} 4, \mathrm{y} 0-\mathrm{y} 5)$ of $1 \mu \mathrm{m}$, with the edges smeared with Gaussian, and the built-in interface trap charge $1 \times 10^{11}$ ions $/ \mathrm{cm}^{2}$; the potential of the $\mathrm{n}$-implant was $0 \mathrm{~V}$ and the backplane $-200 \mathrm{~V}$; the half-width of n-implant (wNsub) was 8 $\mu \mathrm{m}$, the strip pitch of $75 \mu \mathrm{m}$, the thickness of wafer (tSi) 320 $\mu \mathrm{m}$, and the width of p-stop (wPsub) of $6 \mu \mathrm{m}$.

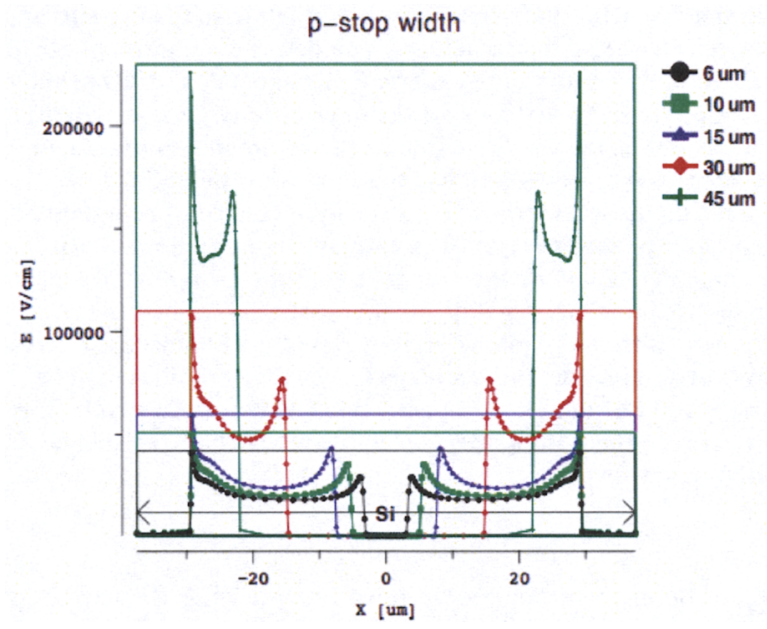

Fig. 5 (a) Electric field strength of the common p-stop structures

$$
\mathrm{p} \text {-stop width }
$$

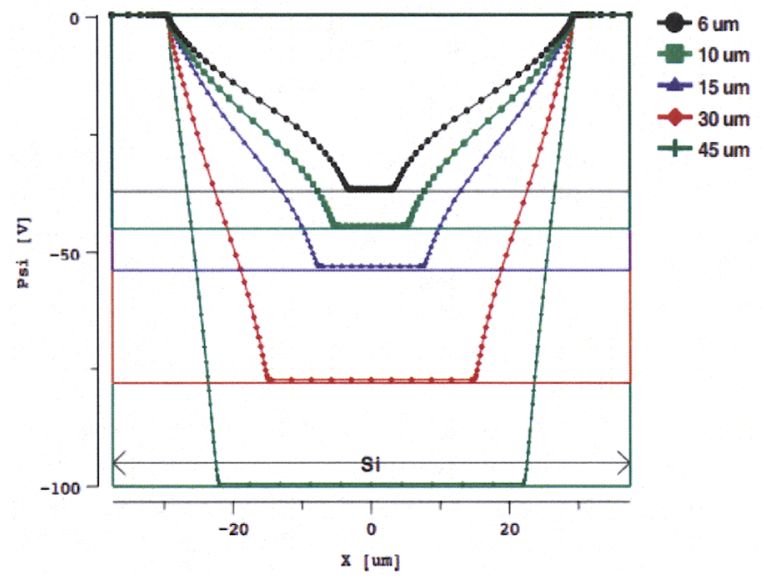

Fig. 5 (b) Electric potential of the common p-stop structures

\section{COMMON P-STOP}

The key to design the robust p-stop structure against the microdischarge is to understand the electric field strength at the implant edges. In the design of the common p-stop structures, the electric fields were evaluated by varying the width of the p-stop to $6,10,15,30$, and $45 \mu \mathrm{m}$. The field strength, E [V/cm], is shown in Fig. 5(a). At the width of 45 $\mu \mathrm{m}$, the largest $\mathrm{E}$ is at the edge of the n-implant and $>200$ $\mathrm{kV} / \mathrm{cm}$ which is already close to the avalanche breakdown voltage of silicon of $300 \mathrm{kV} / \mathrm{cm}$. The least $\mathrm{E}$ was $<50 \mathrm{kV} / \mathrm{cm}$ obtained with the narrowest width. The fundamental of the $\mathrm{E}$ 
is the electric potential of the p-stops as shown in Fig. 5(b): the widest was at about $-100 \mathrm{~V}$ and the narrowest at about -35 $\mathrm{V}$. The wider the width, the $\mathrm{E}$ is enhanced with deeper potential and narrower gap between the n-implant and the pstop (N-P gap). How deep the potential is for the widest width is a surprise as it is about $1 / 2$ of the backplane potential.

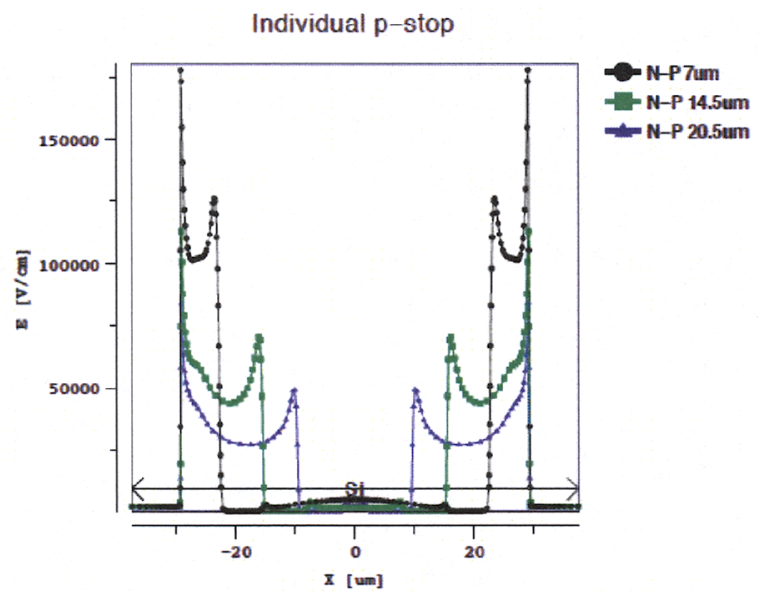

Fig. 6 (a) Electric field strength of the individual p-stop structures

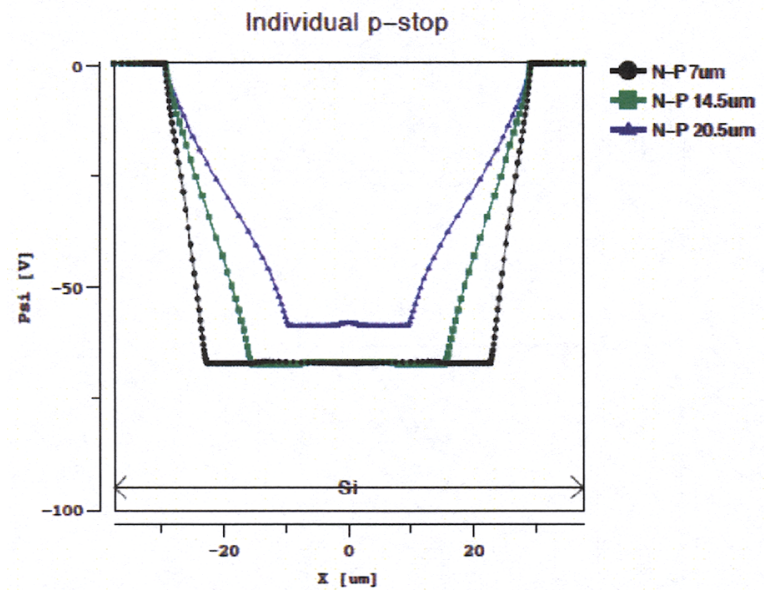

Fig. 6 (b) Electric potential of the individual p-stop structures

\section{INDIVIDUAL P-STOP}

An idea of having a "lighter" p-stop is to split the p-stop and encircle the $n$-implants by individual rings of $p$-stop. A simulation was made by keeping the two p-stops near the nimplants and by eliminating the common p-stop at the center in Fig. 3. In the simulation, the width of p-stop implants was kept constant and the gap between the n-implant and the adjacent p-stop, N-P gap. was varied. The results are shown in Fig. 6(a) and (b). The narrowest N-P gap that is corresponding to the widest width of the common p-stop has a lower E, $<200$ $\mathrm{kV} / \mathrm{cm}$. This is due to a p-stop potential of about $-70 \mathrm{~V}$, shallower than that of the corresponding width of the common p-stop case. We have two surprises: (1) the potential of the bulk between the individual p-stops is only slightly shallower than that of p-stop, thus (p-stop)-(gap)-(p-stop) seems functioning like one large p-stop; (2) the potential of the narrowest N-P gap, i.e., the widest split of two p-stops, is deeper than the widest N-P gap, i.e., the closest split, which is against an expectation that the potential of the widest split would be shallower as the p-stops are closer to the n-implants, i.e., closer to the n-implant potential.

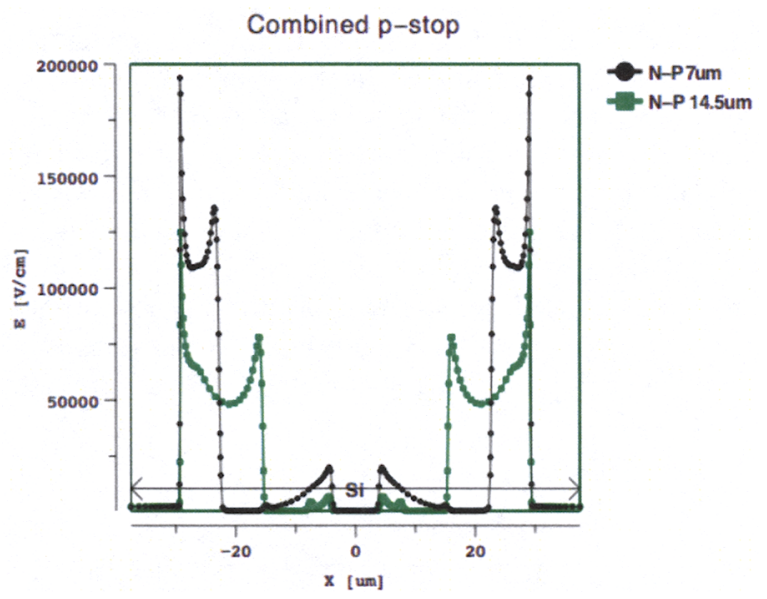

Fig. 7 (a) Electric field strength of the combined p-stop structures

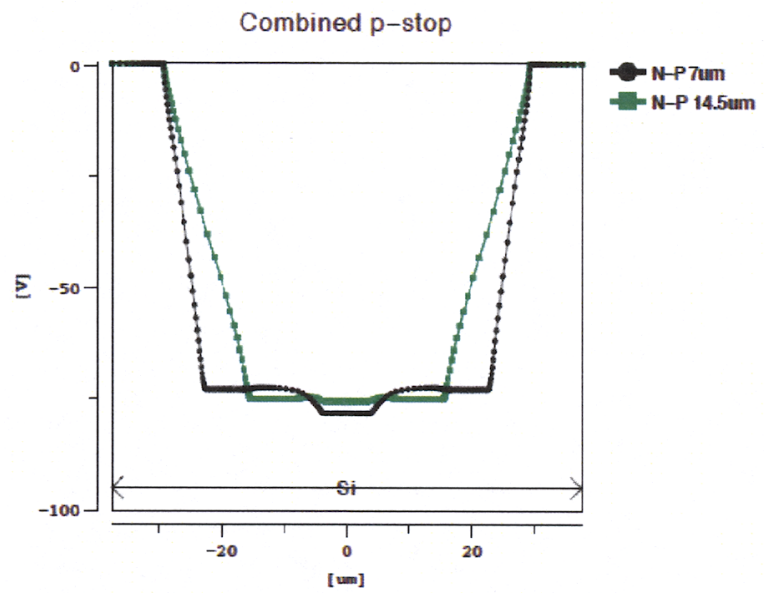

Fig. 7 (b) Electric potential of the combined p-stop structures

\section{COMBINED P-STOP}

The mixture of the common and the individual p-stop is the combined p-stop structure as shown in Fig. 2(c). This structure has been proposed to intercept the accumulation layer outside of the individual p-stop structures which may connect the individual p-stops altogether. This structure may provide "lighter" p-stops around the n-implant, which are separated from the central common p-stop. The width of the common pstop was the narrowest width of the common p-stop cases. The location of the individual p-stops was varied by the N-P gap. The results are shown in Fig. 7(a) and (b). The E's are larger than those of the corresponding individual p-stop cases nor the narrowest common p-stop case, as the potentials were deeper than the corresponding cases. The potential of the individual 
p-stops is as deep as that of the p-stop at the center. The three p-stops behave like one large p-stop, although the p-stop at the center is slightly deeper in potential than the others. The behavior is consistent with the case of individual p-stops, and it was also a surprise against the expectation of the "lighter" $p$ stops that are nearer to the n-implantation.

\section{P-STOP IN ASYMMETRIC POSITION}

As observed in Fig. 2, there could be a case where the pstop is placed asymmetrically between the n-implants, e.g. between the last n-implant strips and the bias ring. The asymmetric cases were simulated with varying the position of the p-stop at the center while the width of the p-stop was kept constant as of the narrowest common p-stop. The results are shown in Fig. 8(a) and (b). The largest E rises as the N-P gap narrows, as expected. A surprise is that the potential of the $p$ stop has changed only a little and is basically the same as that of the symmetric case. Thus, it is the best to place the p-stop symmetrically.

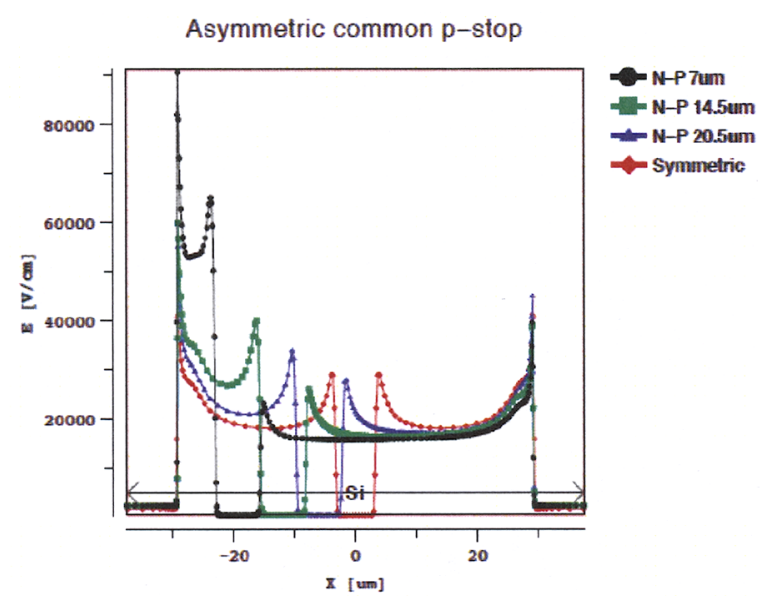

Fig. 8 (a) Electric field strength as a function of strip pitches

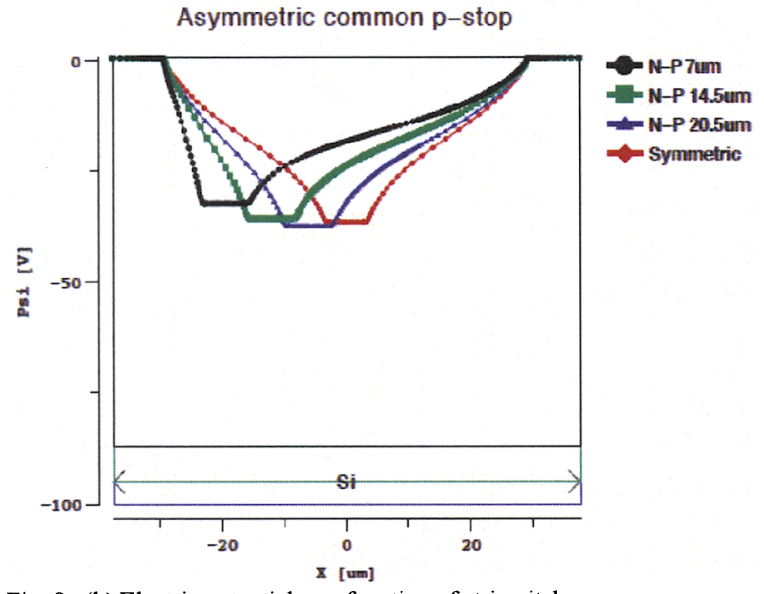

Fig. 8 (b) Electric potential as a function of strip pitches

\section{STRIP PITCH}

Another factor in making the microstrip detector is the pitch of the strips. The strip pitch was varied to $36,50,75,100$, and $150 \mu \mathrm{m}$ for the narrowest common p-stop structure. The electric field strength and the potential are shown in Fig. 9 (a) and (b). The largest electric field strength as a function of strip pitches is shown in Fig. 9 (c). The largest $\mathrm{E}$ rises as the strip pitch narrows below 80 microns, by about $40 \%$ by halving the pitch from 80 to 40 microns; the largest $E$ stays constant as the strip pitch widens above 80 microns. The fundamental is seen in the variation of the electric potential in Fig. 9 (b). This is the interplay of the potential of p-stop and non-linearity of local electric field strength near the strip edges as a function of strip pitch, and more relevantly, the ratio of (p-stop width)/(strip pitch).

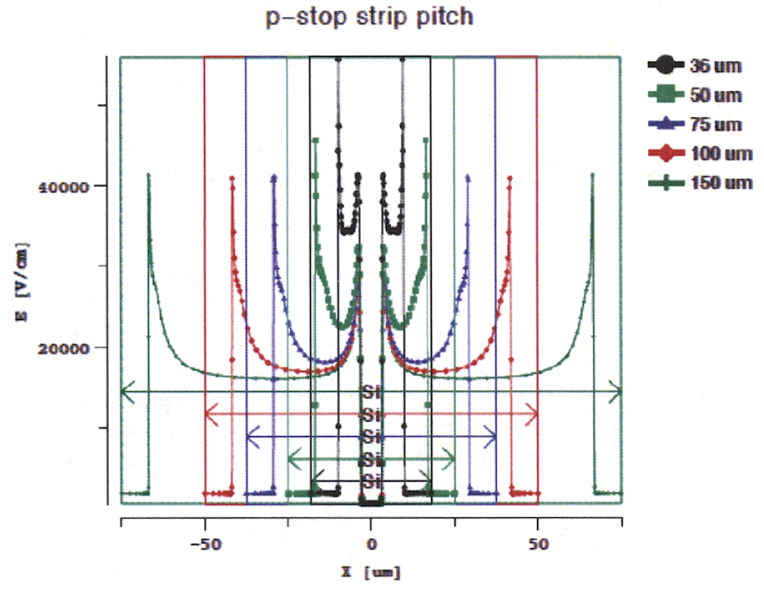

Fig. 9 (a) Electric field strength as a function of strip pitches p-stop strip pitch

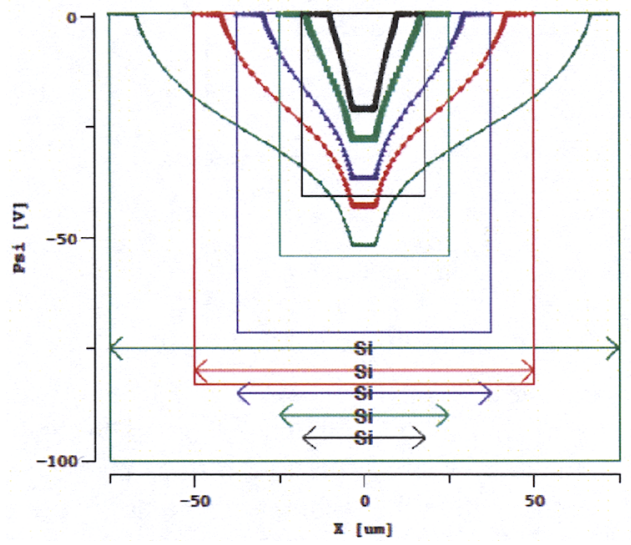

Fig. 9 (b) Electric potential as a function of strip pitches 


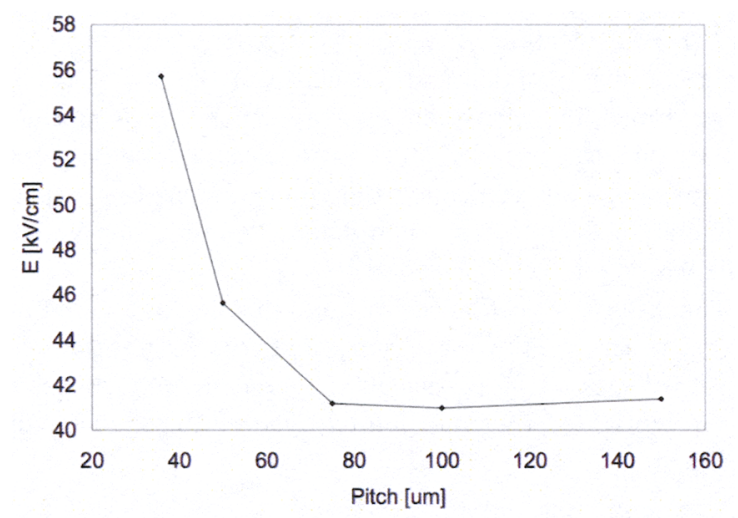

Fig. 9 (c) Largest electric field strength as a function of strip pitches

\section{PunCh-Thru PROTECTION}

For the silicon microstrip sensors whose signals in the implant strips are read out through $\mathrm{AC}$ coupling capacitors, a "punch-thru protection (PTP)" structure is implemented in order to prevent the potential of the strip implants going down more than the breakdown voltage of the insulator of the $\mathrm{AC}$ coupling capacitors. This structure, basically a short distance between the end of the implant strip and the bias ring, is to create a low resistance path in parallel to the bias resisters in case when the silicon bulk is shorted by the deposition of large charges caused by, e.g., beam splash.

Several PTP structures were simulated for the N-N gap of $20 \mu \mathrm{m}$ with a p-stop in between, by varying the voltage of the n-implant of one side. A PTP structure (=default = "Normal") is shown in Fig. 10. Variations to the default structure were to extend the aluminum electrode of the same potential as the nimplant in the right-hand side to cover the p-stop. ("half"), and to cover the full N-N gap, ("full"). Onsets of PTP are shown in Fig. 11 for the PTP structures of Normal. half, and full. and for the two interface-trap charges of $1 \times 10^{11}$ and $1 \times 10^{12} \mathrm{~cm}^{2}$ where the latter could be a case where the surface charge is enhanced by ionization radiation. In Fig. 11, the legends are ("Normal", "half", "full") x $1 \times 10^{11}=$ (black, blue, green), and ("Normal", "half". "full") x $1 \times 10^{12}=$ (green, red, violet). The amount of interface-trap charge affects the onset voltage the most. The extended coverage also helps to reduce the onset voltage, but the difference due to the half or full coverage is small or none for the larger interface-trap charges.

A comparison was made for the PTP with the p-spray method with a dopant concentration of $2 \times 10^{12}$ ions $/ \mathrm{cm}^{2}$. Onsets of the PTP are shown in Fig. 12 for the N-N gap of 20 and $12 \mu \mathrm{m}$. For the same N-N gap of $20 \mu \mathrm{m}$, the onset voltages are similar for the PTP with p-stop and p-spray, thus the onset is basically determined by the $\mathrm{N}-\mathrm{N}$ gap distance. The p-spray of N-N gap of $12 \mu \mathrm{m}$ shows the onset at a lower voltage. This is due to the larger $\mathrm{E}$ in the N-N gap of $12 \mu \mathrm{m}$, $>100 \mathrm{kV} / \mathrm{cm}$, than that of $20 \mu \mathrm{m}$, as shown in Fig. 13 .
NormalBRt1e11\#1_dis.dists50.E

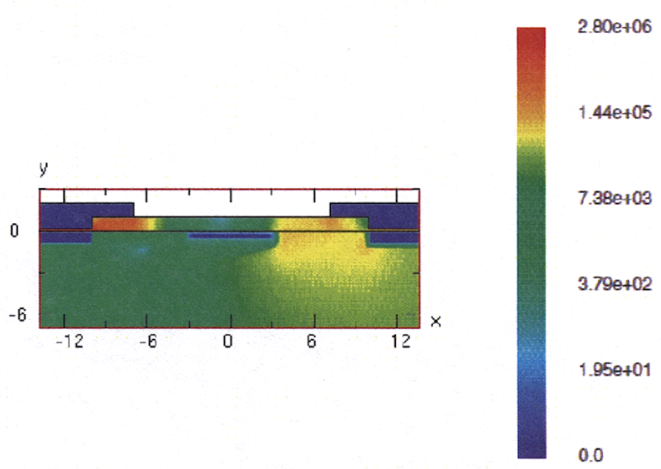

Fig. 10 Geometry of PTP with p-stop, with an electric field where the voltage of $50 \mathrm{~V}$ is put on the n-implant in the left side

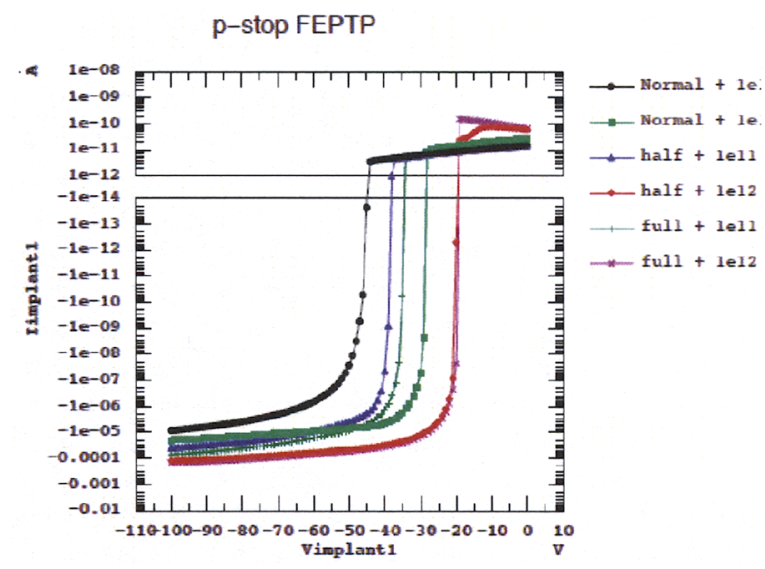

Fig. 11 Punch-thru current over $p$-stop as a function of n-implant voltage of a side

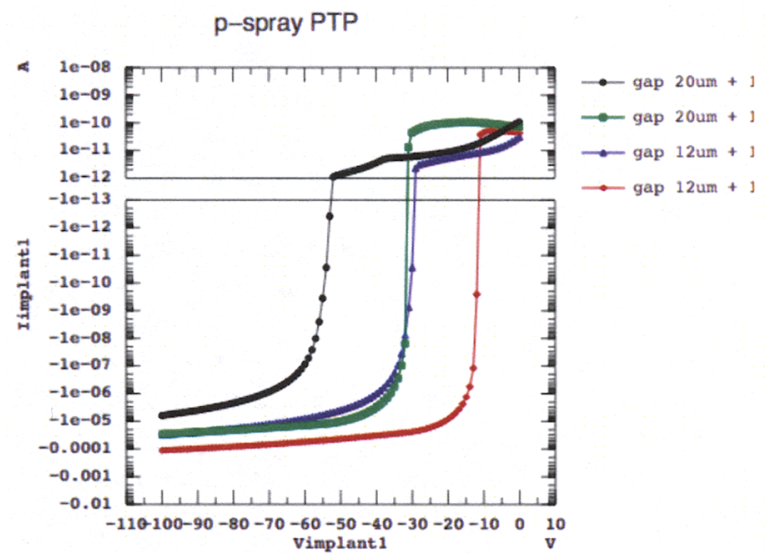

Fig. 12 Punch-thru current over $\mathrm{p}$-spray as a function of n-implant voltage of a side 


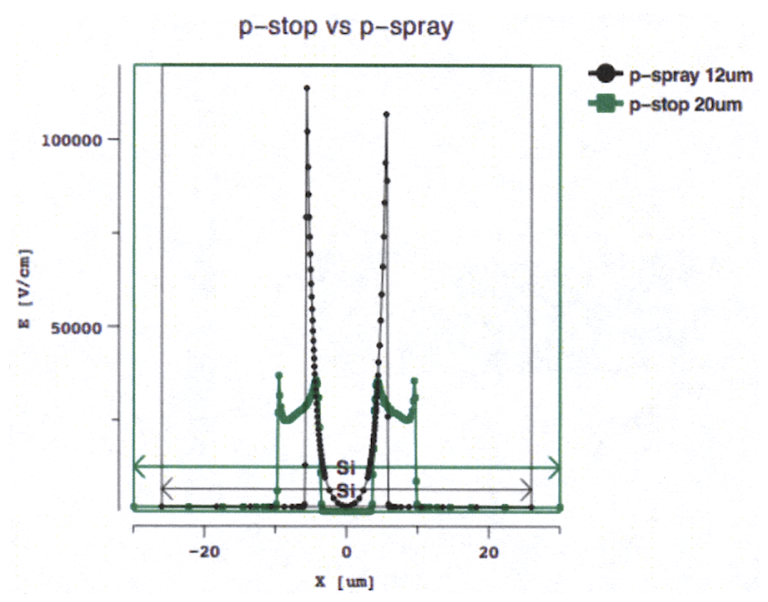

Fig. 13 Electric field strength of p-stop and p-spray with n-implant voltage at $0 \mathrm{~V}$

\section{SUMmary}

In the SLHC where the luminosity is to be increased 10 -fold, from $10^{34}$ to $10^{35} \mathrm{~cm}^{-2} \mathrm{~s}^{-1}$, the fluence of particles is expected to be $1 \times 10^{15} 1-\mathrm{MeV}$ neutron-equivalent $/ \mathrm{cm}^{2}$ at a radius of around $30 \mathrm{~cm}$ towards the end of experiment. A new type of radiation tolerant silicon microstrip sensor is required and a novel $n-i n-p$ sensor is being developed. In order to achieve isolation of the strips in the n-side, a structure to intercept the electron accumulation layer due to the positively charged interface between the $\mathrm{Si}$ and $\mathrm{SiO}_{2}$ has to be implemented, which is achieved by the p-type implantation, p-stop structures, in between the $n$-implant strips.

The difficulty in designing the radiation-tolerant silicon microstrip sensors is to expel the onset of microdischarge, i.e., a steep rise of leakage current, above the maximum operation voltage. The difficulty is enhanced in the $n$-in-p sensors, with the existence of $p-n$ junction in the n-strips and the existence of p-stop structures. The sophisticated device simulation program available in the semiconductor industry, ENEXSS in our case, enabled to understand the electric fields associated with the p-stop structures and to optimize the design to be robust against the microdischarge.

Three types of p-stop structures, common p-stop, individual p-stop, and combined p-stop, were simulated, by varying the p-stop parameters, such as width, N-P gap, location, etc. What we have leaned are: the potential of the p-stop is the fundamental; in the common p-stop structures, the narrowest p-stop width has the shallowest potential and generating the least of the largest electric field strength (Emax). The potentials of the split p-stops and the p-bulk in between, (individual p-stop), or the p-stop in between, (combined $\mathrm{p}$ stop), have shallower potential than that of the same total width of common p-stop, but not as shallow as the common pstop of the single width of split p-stops; split p-stops may work as a single wide p-stop effectively, thus, the Emax is larger than that of the narrowest common p-stop as the width of $\mathrm{p}$-stops altogether is wider than the narrowest common $\mathrm{p}$ stop. The potential of the split $\mathrm{p}$-stops near to the n-implant does not have a potential closer to that of the n-implant. In the asymmetric location of p-stops, the potential of the p-stops does not change much and the narrower the N-P gap is, the larger Emax, thus the symmetric case has the least Emax. The Emax increases as the strip pitch decreases less than 80 microns but stays the same as the pitch widens larger than 80 microns, in this geometry and parameters of p-stop width, pbulk wafer, pitch etc

Punch-thru protection (PTP) was simulated as a variation of p-stop structures. In comparison with the p-spray structure, the onset voltage of PTP is basically governed by the gap between the $n$-implants, $\mathrm{N}-\mathrm{N}$ gap, even with the existence of $\mathrm{p}$-stop in between.

\section{ACKNOWLEDGMENTS}

We acknowledge the support by ATLAS SCT upgrade sensor collaboration for the development of the $n$-in-p silicon microstrip sensor and by the detector development program by KEK for the usage of the TCAD program, ENEXSS.

\section{REFERENCES}

[1] A. Ahmad et al, 'The silicon microstrip sensors of the ATLAS semiconductor tracker', Nucl. Instr. Meth. A 578 (2007) 98-118

[2] Y. Unno, 'Silicon sensor development for the ATLAS upgrade for SLHC', Nucl. Instr. Meth. A569 (2006) 41-47

[3] Stanford Technology CAD home page, http://wwwtcad.stanford.edu/tcad.html

[4] ENEXSS, TCAD International Inc., http://www.tcadinternational.com/english/product/index.html 